\title{
Flexible Mixed-Initiative Dialogue Management using Concept-Level Confidence Measures of Speech Recognizer Output
}

\author{
Kazunori Komatani and Tatsuya Kawahara \\ Graduate School of Informatics, Kyoto University \\ Kyoto 606-8501, Japan \\ \{komatani, kawahara\}(1)kuis.kyoto-u.ac.jp
}

\begin{abstract}
We present a method to realize flexible mixedinitiat,ive dialogue, in which the system can make effective confirmation and guidance nsing concept-level confidence measures (CMs) derived from speech recognizer output in order to handle speech recognition errors. We define two concept-mevel CMs, which are on contentwords and on semantic-attributes, using 10-best outputs of the speech recognimer and parsing with phrase-level grammars. Content-word CM is useful for selecting plausible interpretations. less confictent interpretations are given to confirmation process. The strategy inproved the interpretation accuracy by $11.5 \%$. Moreover, the semantic-attribute CM is used to estimate user's intention and generates system-initiative guidances even when successful interpretation is noti obtained.
\end{abstract}

\section{Introduction}

In a spoken dialogue system, it frequently occurs that the system incorrectly recognizes user utterances and the user makes expressions the system has not expected. These problems are essentially incvitable in handling the natural language by computers, even if vocabulary and grammar of the system are tuncel. This lack of robustness is one of the reason why spoken dialogue systems have not been widely deployed.

In order to realize a robust spoken dialogue system, it is inevitable to handle speech recognition errors. To suppress recognition errors, system-initiative dialogue is effective. But it can be adopted only in a simple task. For instance, the form-filling task can be realized by a simple strategy where the system asks a user the slot values in a fixed order. In such a systeminitiated interaction, the recognizer easily narrows down the vocabulary of the next user's ut- torance, thens the recognition gets easior.

On the other hand, in more complicated task such as information retrieval, the vocabulary of the next utterance cannot be limited on all occasions, because the user should be able to input the values in various orders based on his preference. Therefore, without imposing a rigid template upon the user, the system must behave appropriately even when speech recognizer output contains some crrors.

Obviously, making confirmation is effective to avoid misunderstandings caused by speech recognition errors. However, when confirmations are made for every utterance, the dialogue will become too redundant and consecuuently troublesome for users. Previous works have shown that confirmation strategy should be decided according to the frepuency of speech recognition errors, using mathematical formula (Niimi and Kobayashi, 1996) and using computer-to-computer simulation (Watanabe of al., 1998). 'These works assume fixed performance (averaged speech recognition accuracy) in whole dialogue with any speakers. For: flexible dialogue managenent, however the confirmation strategy must be dynamically changed based on the individual utterances. For instance, we human make confirmation only when we are not confident. Similarly, confidence measures (CMs) of every speech recognition output should be modeled as a criterion to control dialogue management.

CMs have been calculated in previous works using transcripts and various knowledge sources (Litman et al., 1999) (Pao et al., 1998). For more flexible interaction, it is desirable that CMs are defined on each word rather than whole sentence, because the system can handle only umreliable portions of an utterance instead of accepting/rejecting whole sentence. 
In this paper, we propose two concept-level CMs that are on content-word level and on semantic-attribute level for every content word. Because the CMs are defined using only speech recognizer output, they can be computed in real time. The system can make efficient confirmation and effective guidance according to the CMs. Even when successful interpretation is not obtained on content-word level, the system generates system-initiative guidances based on the semantic-attribute level, which lead the next uscr's utterance to successful interpretation.

\section{Definition of Confidence Measures (CMs)}

Confidence Measures (CMs) have been studied for utterance verification that verifies specch recognition result as a post-processing (Kawahara et al., 1998). Since an automatic speech recognition is a process finding a sentence hypothesis with the maximum likelihood for an input specch, some measures are needed in order to distinguish a correct recognition result from incorrect one. In this section, we describe definition of two lovel CMs which are on content-words and on semantic-attributes, using 10-best output of the speech recognizer and parsing with phrase-level grammars.

\subsection{Definition of CM for Content Word}

In the speech recognition process, both acoustic: probability and linguistic probability of words are multiplied (summed up in log-scale) over a sentence, and the sequence having maximum likclihood is obtained by a search algorithm. A score of sentence derived from the speech recognizer is log-scaled likelihood of a hypothesis sequence. We use a grammar-based speech recognizer Julian (Lee et al., 1999), which was developed in our laboratory. It correctly obtains the N-best candidates and their scores by using A* search algorithm.

Using the scores of these N-best candidates, we calculate content-word CMs as below. The content words are extracted by parsing with phrase-level grammars that are used in speech recognition process. In this paper, we set $N=$ 10 after we examined various values of $N$ as the number of computed candidates ${ }^{\perp}$.

\footnotetext{
${ }^{1}$ Even if we set $N$ larger than 10 , the scores of $i$-th hypotheses $(i>10)$ are too small to affect resulting CMs.
}

First, each $i$-th score is multiplied by a factor $\alpha(\alpha<1)$. This factor smoothes the difference of N-best scores to get adequately distributed CMs. Because the distribution of the absolute values is different among kinds of statistical acoustic model (monophone, triphone, and so on), different values must be used. The value of $\alpha$ is examined in the preliminary experiment. In this paper, we set $\alpha=0.05$ when using triphone model as acoustic model. Next, they are transformed from log-scaled value $\left(\alpha \cdot\right.$ scaled $\left._{i}\right)$ to probability dimension by taking its exponential, and calculate a posteriori probability for each $i$-th candidate (Bouwman et al., 1999).

$$
p_{i}=\frac{e^{\alpha \cdot \text { scaled }_{i}}}{\sum_{j=1}^{n} e^{\alpha \cdot \text { scaled }_{j}}}
$$

This $p_{i}$ represents a posteriori probability of the $i$-th sentence hypothesis.

Then, we compute a posteriori probability for a word. If the $i$-th sentence contains a word $w$, let $\delta_{w, i}=1$, and 0 otherwisc. A posteriori probability that a word $w$ is contained $\left(p_{w}\right)$ is derived as summation of a posteriori probabilities of sentences that contain the word.

$$
p_{w}=\sum_{i=1}^{n} p_{i} \cdot \delta_{w, i}
$$

We define this $p_{w}$ as the content-word CM $\left(C M_{w}\right)$. This $C M_{w}$ is calculated for every content word. Intuitively, words that appear many times in N-best hypotheses get high CMs, and frequently substituted ones in N-best hypotheses are judged as unreliable.

In Figure 1, we show an example in $C M_{w}$ calculation with recognizer outputs ( $i$-th recognized candidates and their a postcriori probabilities) for an utterance "Futaishisetsu ni resutoran no aru yado (Tell me hotels with restaurant facility.)". It can be observed that a correct content word 'restaurant as facility' gets a high $\mathrm{CM}$ value $\left(C M_{w}=1\right)$. The others, which are incorrectly recognized, get low $\mathrm{CMs}$, and shall be rejected.

\subsection{CM for Semantic Attribute}

A concept category is semantic attribute assigned to content words, and it is identificd by parsing with phrase-level grammars that are used in speech recognition process and represented with Finite State Automata (FSA). Since 


\begin{tabular}{|c|c|c|}
\hline & Recognition candidates & $p_{i}$ \\
\hline 1 & $\begin{array}{l}\text { an shisetsu ni resutoran no kayacho } \\
\text { with restaurant facility / Kayacho(location) }\end{array}$ & .24 \\
\hline 2 & $\begin{array}{l}\text { a shisetsu ni resutoran no katsura no } \\
\text { with restaurant facility / Katsura(location) }\end{array}$ & .21 \\
\hline 3 & $\begin{array}{l}\text { a shisetsu ni resutoran no hamigamo } \\
\text { with restaurant facility / Kamigamo(location) }\end{array}$ & .20 \\
\hline 4 & $\begin{array}{l}<\mathrm{g}>\text { shisetsu ni resutoran no kayacho } \\
\text { with restaurant, facility / Kayacho(location) }\end{array}$ & .08 \\
\hline 5 & $\begin{array}{l}\langle\mathrm{g}\rangle \text { shisetsu ni resutoran no katsurra } \\
\text { with restaurant facility / Katsura(location) }\end{array}$ & .08 \\
\hline 6 & $\begin{array}{l}\langle\mathrm{g}\rangle \text { shisetsu ni resutoran no kamigamo } \\
\text { with restaurant facility / Kamigamo(location) }\end{array}$ & .06 \\
\hline 7 & $\begin{array}{l}\text { al shisetsu ni resutoran no kafe } \\
\text { with restaurant facility / cafo(facility) }\end{array}$ & .05 \\
\hline 8 & $\begin{array}{l}\langle\mathrm{g}\rangle \text { shisetsu ni resutoran no kafe } \\
\text { with restaurant facility / cafe(facility) }\end{array}$ & .02 \\
\hline 9 & $\begin{array}{l}\langle\mathrm{g}\rangle \text { setsubi wo resutoran no kayacho } \\
\text { with restaurant facility / Kayacho(location) }\end{array}$ & .01 \\
\hline & $\begin{array}{l}\langle\mathrm{g}\rangle \text { setsubi wo resutoran no katsura no } \\
\text { with restaurant lacility / Katsura(location) }\end{array}$ & .01 \\
\hline
\end{tabular}

$\langle\mathrm{g}\rangle$ : filler model

\begin{tabular}{c|rrl}
$C M_{u}$ & (content word) & $@$ & (scmantic attribute) \\
\hline 1 & restaurant & $\bullet$ & facility \\
0.33 & Kayacho & 0 & location \\
0.33 & Katsura & 0 & location \\
0.25 & Kamigamo & 0 & location \\
0.07 & cafe & $\bigotimes$ & facility
\end{tabular}

Figure 1: Example of content-word CM $\left(C M_{w}\right)$

these FSAs are classified into concept categories beforchand, we can antomatically derive the concept categories of words by parsing with these grammars. In our hotel query task, there are seven concept categoriess such as 'Jocation', 'facility' and so on.

For this concept catcgory, we also define scmantic-attribute CMs $\left(C M_{c}\right)$ as follows. First, we calculate a posteriori probabilities of $\mathrm{N}$-best sentences in the same way of computing content-word CM. If a concept category $c$ is contained in the $i$-th sentence, let $\delta_{c, i}=1$, and 0 otherwise. The probability that a concept category $c$ is correct $\left(p_{c}\right)$ is derived as below.

$$
p_{c}=\sum_{i=1}^{n} p_{i} \cdot \delta_{c, i}
$$

Wo define this $p_{c}$ as semantic-attribute CM $\left(C M_{c}\right)$. This $C M_{c}$ estimates which category the user refers to and is used to generate eflective guidances.

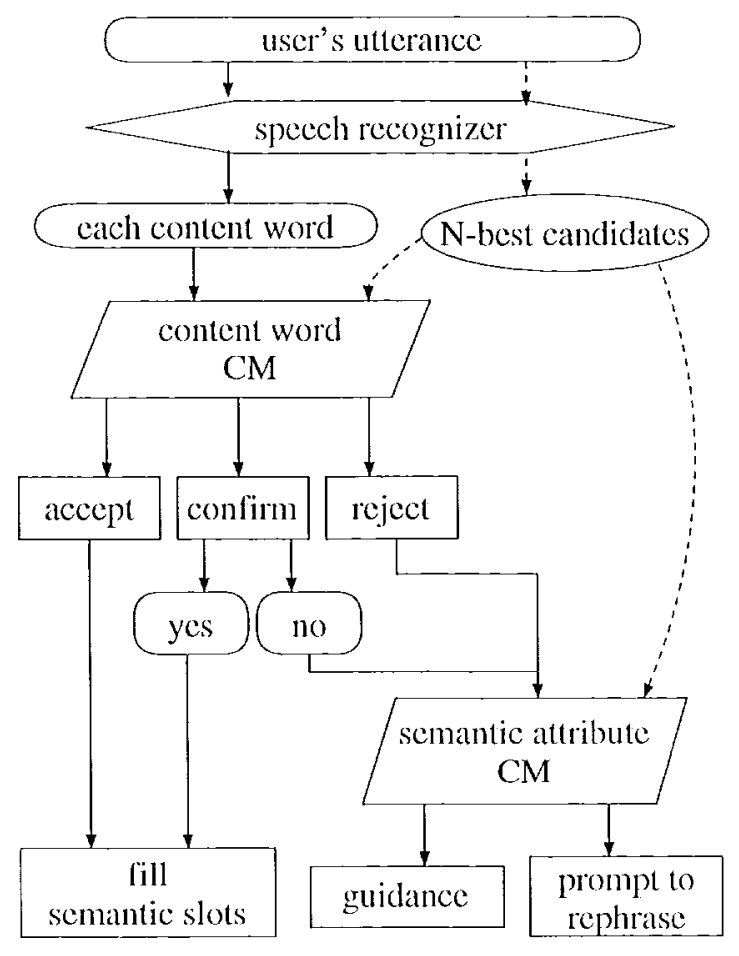

Figure 2: Overview of our strategy

\section{Mixed-initiative Dialogue Strategy using CMs}

J'here are a lot of systems that have adopted a mixed-initiative strategy (Sturm of al., 1999)(Goddeau ot al., 1996)(Bennacef of al., 1996). It has several advantages. As the systems do not impose rigid system-initiated templates, the user can input values he has in mind directly, thus the dialogue becomes more natural. In conventional systems, the systeminitialed utterances are considered only when semantic ambiguity occurs. But in order to realize robust interaction, the system should make confirmations to remove recognition errors and generate guidances to lead next user's utterance to successful interpretation. In this section, we describe how to generate the system-initiated utterances to deal with recognition errors. An overview of our strategy is shown in Figure 2.

\subsection{Making Effective Confirmations}

Confidence Measure (CM) is useful in selecting reliable candidates and controlling confirmation strategy. By setting two thresholds $\theta_{1}, \theta_{2}\left(\theta_{1}>\right.$ $\left.0_{2}\right)$ on content-word CM $\left(C M_{u}\right)$, we provide the confirmation stratcgy as follows. 
- $C M_{w}>0_{1}$

$\rightarrow$ accept the hypothesis

- $\theta_{1} \geq C M_{w}>\theta_{2}$

$\rightarrow$ make confirmation to the user

"Did you say ...?"

- $\theta_{2} \geq C M_{w}$

$\rightarrow$ reject the hypothesis

The threshold $\theta_{1}$ is used to judge whether the hypothesis is accepted or should be confirmed, and the threshold $\theta_{2}$ is used to judge whether it is rejected.

Because $C M_{w}$ is defined for every content word, judgment among acceptance, confirmation, or: rejection is made for cvery content word when one utterance contains several content words. Suppose in a single utterance, one word has $C M_{w}$ between $\theta_{1}$ and $\theta_{z}$ and the other: has below $\theta_{2}$, the former is given to confirmation process, and the latter is rejected. Only if all content words are rejected, the system will prompt the user to utter again. By accepting confident words and rejecting unreliable candidates, this strategy avoids redundant confirmations and focuses on necessary confirmation.

Wo optimize these thresholds $\theta_{1}, \theta_{2}$ considering the false acceptance (FA) and the false rejection (FR) using real data.

Moreover, the system should confirm using task-level knowledge. It is not usual that users change the already specified slot values. Thus, recognition results that overwrite filled slots are likely to be errors, even though its $C M_{w}$ is high. By making confirmations in such a situation, it is expected that false acceptance (FA) is suppressed.

\subsection{Generating System-Initiated Guidances}

It is necessary to guide the users to recover from recognition errors. Especially for novice users, it is often effective to instruct acceptable slots of the system. It will be helpful that the system generates a guidance about the acceptable slots when the user is silent without carrying out the dialogue.

The system-initiated guidances are also effective when recognition does not go well. Even when any successful output of content words is not obtained, the system can generate effective guidances based on the semantic attribute with utterance: "shozai ga oosakafu no yado" (hotels located in Osaka pref.)

correct: Osaka-pref.@location

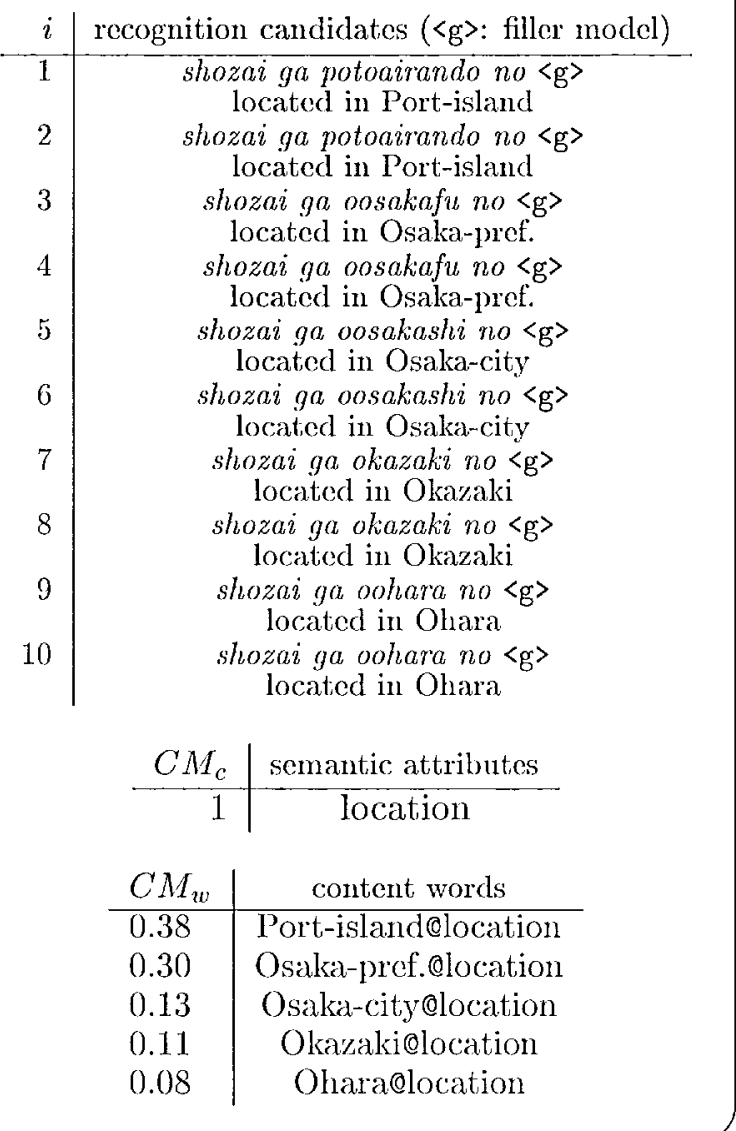

Figure 3: Example of high semantic atitribute confidence in spite of low word confidence

high confidence. An example is shown in Figure 3. In this example, all the 10-best candidates are concerning a name of place but their $C M_{w}$ values are lower than the threshold $\left(\theta_{2}\right)$. As a result, any word will be neither accepted nor confirmed. In this casc, rather than rejecting the whole sentence and telling the user "Please say again", it is better to guide the user based on the attribute having high $C M_{c}$, such as "Which city is your destination?". This guidance enables the system to narrow down the vocabulary of the next user's utterance and to reduce the recognition difficulty. It will consequently lead next user's utterance to successful interpretation.

When recognition on a content word does not 
go well repeatedly in spite of high semanticattribute CM, it is reasoned that the content, word may be out-of-vocabulary. In such a case, the system should change the question. For example, if an utterance contains an out-ofvocabulary word and its somantic-attribute is inferred as "location", the system canl make guidance, "Please specify with the name of prefecture", which will lead the next uscr's utterance into the system's vocabulary.

\section{Experimental Evaluation}

\subsection{Task and Data}

Wo evaluate our method on the hotel query task. We collected 120 minultes speech datia by 24 novice users by using the prototype system with GUI (Figure 4) (Kawahara et al., 1999). The users were given simple instruction beforehand on the system's task, retrievable items, how to cancel input values, and so on. The data is segmented into 705 utterances, with a pautse of 1.25 seconds. The vocabulary of the system contains 982 words, and the number of database records is 2040 .

Out of 705 utterances, 124 ut terances $(17.6 \%)$ are beyond the system's capability, namely they are out-of-vocabulary, out-of-grammar, out-oftask, or fragment of utterance. In following experiments, we evaluate the system performance? using all cata inchuding these macecetable utterances in order to evaluate how the system can reject unexpected utterances appropriately as woll as recognize normal utterances correctly.

\subsection{Thresholds to Make Confirmations}

In section 3.1, we presented confirmation stratcgy by setting two thresholds $\theta_{1}, \theta_{2}\left(\theta_{1}>\theta_{2}\right)$ for content-word CM $\left(C M_{w}\right)$. We optinnize these threshold values using the collected datia. We count errors not by the utterance but by the content-word (slot). The number of slots is 804 .

The threshold $\theta_{1}$ decides between acceptance and confirmation. The value of $\theta_{1}$ should be determined considering both the ratio of incorrectly accepting recognition errors (False Acceptance; $F \Lambda$ ) and the ratio of slots that are not filled with correct values (Slot Error; SErr). Namely, FA and Sterr are defined as the complements of precision and recall rate of the output, respectively.

$$
\begin{gathered}
I P A=\frac{\# \text { of incorrectly accepted words }}{\# \text { of accepted words }} \\
\text { SErr }=1-\frac{\# \text { of correctly accepted words }}{\# \text { of all correct words }}
\end{gathered}
$$

After experimental optimization to minimize $\mathrm{FA}+\mathrm{SEST}$, we derive a value of $\theta_{1}$ as 0.9 .

Similarly, the threshold $\theta_{2}$ decides confirmation and rejection. The value of $\mathrm{O}_{2}$ should be decided considering both the ratio of incorrectly rejecting content; words (False Rejection; FR) and the ratio of accepting recognition errors into the confirmation process (conditional False Acceptance; ( $\mathrm{P} \Lambda$ ).

$$
F R=\frac{\# \text { of incorrectly rejected words }}{\# \text { of all rejected words }}
$$

If we set the threshold $O_{2}$ lower, FR decreases and correspondingly $\mathrm{CFA}$ increases, which moans that more candidates are obtained but more confirmations are needed. By minimiring liR $+\mathrm{erA}$, we derive a value of $\theta_{2}$ as 0.6 .

\subsection{Comparison with Conventional Methods}

In many conventional spoken dialogue systems, only 1-best candidate of a speech recognizer output is used in the subsecuent procossings. We compare our mothod with a conventional method that uses only 1-best candidate in interpretation accuracy. 'The result is shown in Table 1.

In the 'no confirmation' strategy, the lypotheses are classified by a single threshold (0) into either the aceepted or the rejected. Namely, content words having $C M_{w}$ over threshold 0 are accepted, and otherwise simply rejected. In this case, a threshold value of $\theta$ is set to 0.9 that gives minimum $\mathrm{FA}+\mathrm{SEEr}$. In the 'with confirmation' strategy, the proposed confirmation strategy is adopted using $\theta_{1}$ and $O_{2}$. We set $\theta_{1}=0.9$ and $\theta_{2}=0.6$. The 'FA+SErr' in Table 1 means $F \Lambda\left(O_{1}\right)+\operatorname{SErr}\left(O_{2}\right)$, on the assimption that the confirmed phrases are correctly either acecepted or rejected. Wo regard this assumption as appropriate, bocause users tend to answer "yes' simply to express their affirmation (Hockey et al., 1997), so the system can distinguish affirmative answer and negative one by grasping simple 'yes' utterances correctly. 


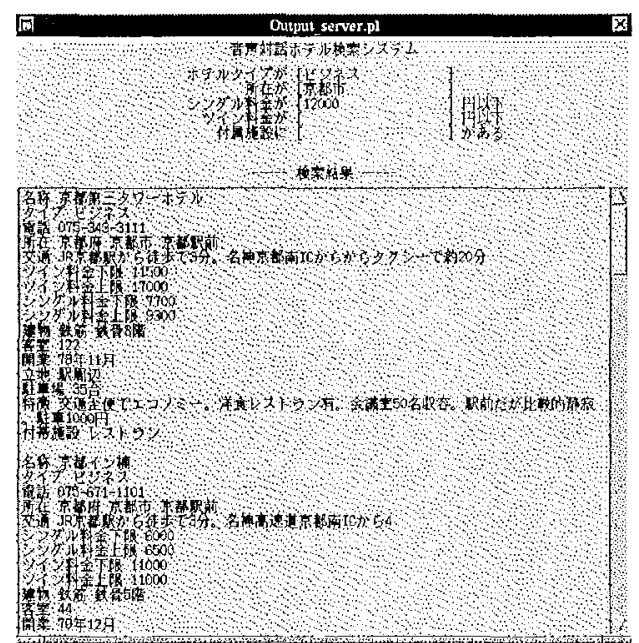

(a) A real system in Japanese

\section{Hotel Accommodation Search}

$\begin{array}{ll}\text { hotel type is } & \text { Japanese-style } \\ \text { location is } & \text { downtown Kyoto }\end{array}$

room rate is less than 10,000 yen

These are query results :

(b) Upper portion translated in English

Figure 4: An outlook of GUI (Graphical User Interface)

Table 1: Comparison of methods

\begin{tabular}{c||c|c|c}
\hline & FA+SErr & FA & SErr \\
\hline only 1st candidate & 51.5 & 27.6 & 23.9 \\
no confirmation & 46.1 & 14.8 & 31.3 \\
with confirmation & 40.0 & 14.8 & 25.2 \\
\hline
\end{tabular}

FA: ratio of incorrectly accepting recognition crrors

SErr: ratio of slots that are not filled with correct values

As shown in Table 1, interpretation accuracy is improved by $5.4 \%$ in the 'no confirmation' strategy compared with the conventional method. And 'with confirmation' strategy, we achieve $11.5 \%$ improvement in total. This result proves that our method successfully climinates recognition crrors.

By making confirmation, the interaction becomes robust, but accordingly the number of whole utterances increases. If all candidates having $C M_{w}$ under $\theta_{1}$ are given to confirmation process without setting $\theta_{2}, 332$ vain confirmation for incorrect contents are generated out of 400 candidates. By setting $\theta_{2}, 102$ candidates having $C M_{w}$ between $\theta_{1}$ and $\theta_{2}$ are confirmed, and the number of incorrect confirmations is suppressed to 53 . Namely, the ratio of correct hypotheses and incorrect ones being confirmed are almost equal. This result shows indistinct candidates are given to confirmation process whereas scarcely confident candidates are rejected.

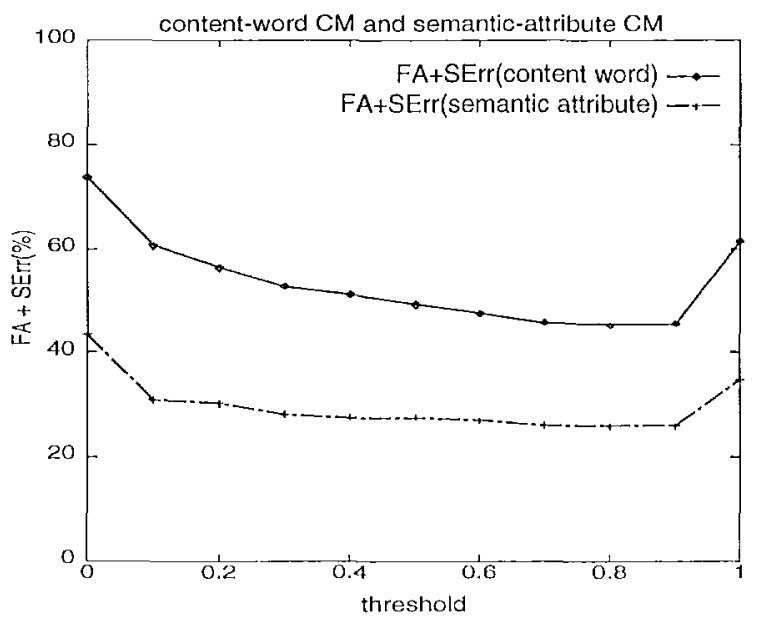

Figure 5: Porformance of the two CMs

\subsection{Effectiveness of Semantic-Attribute CM}

In Figure 5, the relationship between contentword CM and semantic-attribute CM is shown. It is observed that semantic-attribute CMs are estimated more correctly than content-word CMs. Therefore, even when successful interpretation is not obtained from content-word CMs, semantic-attribute can be estimated correctly.

In experimental data, there are 148 slots $^{2}$ that are rejected by content-word CMs. It is also observed that $52 \%$ of semantic-attributes

\footnotetext{
${ }^{2}$ Out-of-vocabulary and out-of-grammar utterances are included in their phrases.
} 
with $C M_{c}$ over 0.9 is correct. Such slots amount to 34. Namely, our system can generate effective guidances against $23 \%(34 / 148)$ of utterances that had been only rejected in conventional methods.

\section{Conclusion}

We present dialoguo management using two concept-level CMs in order to realize robust interaction. Tho content-word CM provides a criterion to decide whether an interpretation should be accepted, confirmed, or rejected. This strategy is realized by setting two thresholds that are optimized balancing false acceptance and false rejection. The interpretation crror $(\mathrm{F} \Lambda+\mathrm{SErr})$ is reduced by $5.4 \%$ with no confirmation and by $1.1 .5 \%$ with confirmations. Moreover, we definc CM on semantic; attributes, and propose a new method to gencrate eflective guidances. The concept-based confidence mnasure realizes flexible mixed-initiative dialogue in which the system can make offective confirmation and guidance by estimating nser's intention.

\section{References}

S. Bemancef, I. Devillers; S. Rosset, and L. Lamnel. 1996. Dialog in the RAILTEL telephone-based system. In Proc. Int'l Conf. on Spolien Language Processing.

G. Bouwnan, J. Sturm, and I. Boves. 1999. Incorporating confidence measures in the Dutch train timetable information system developed in the AIRISE project. In Proc. ICASSP.

D. Goddean, H. Meng, J. Polifroni, S. Sonoff, and S. Busayapongchai. 1996. $\Lambda$ form-based dialogue manager for spoken language applications. In Proc. Int'l Conf. on Spoken Isanguage Processing.

B. A. Hockey, D. Rossen-Knill, B. Spejewski, M. Stone, and S. Isard. 1997. Can you predict responses to yes/no questions? yes,no,and stuff. In Proc. LUROSPELCH'97.

T. Kawahara, C.-H. Lec, and B.-H. Juang. 1998. Flexible specch 11nderstanding based on combined key-phrase detection and verification. IEEE Trans. on Speech and Audio Processing, 6(6):558-568.

T'. Kawahara, K. Tanaka, and S. Doshita. 1999. Domain-independent platform of spoken dialogue interfaces for information query. In
Proc. ESCA workshop on Interactive Dialogue in Multi-Modal Systems, pages 6972.

A. Lee, T. Kawahara, and S. Doshita. 1999. Large vocabulary continuous specch recognition parser based on $A^{*}$ search using grammar catcgory category-pair constraint (in Japanese). Trans. Information Processing Socicty of Japan, 40(4):1374-1382.

D. J. Litman, M. A. Walker, and M. S. Koarms. 1999. Antomatic detection of poor speech recognition at the dialogue level. In proc. of 37th. Annual Meeting of the ACL.

Y. Niimi and Y. Kobayashi. 1996. A dialog control strategy based on the reliability of speech recognition. In Proc. Int'l Conf. on Spoken, Language Processing.

C. Pao, P. Schmid, and J. Glass. 1998. Confidence scoring for spech understanding systicms. In Proc. Int'l Conf. on Spolen Language Processing.

J. Sturm, L. Os, and L. Boves. 1999. Issues in spoken dialogue systems: Experiences with the Dutch ARISE system. In Proc. of IDSCA IDS'gg Workshop.

T. Watanabe, M. Araki, and S. Doshita. 1998. Evaluating dialogue strategies under communication emors using computer-to-computer simulation. Trans. of IEICE, Info \& Syst., 1) $81-\mathrm{D}(9): 1025 \cdots 1033$. 